\section{PA-115 FUNCTIONAL COMMUNICATION IN MULTISITE, MULTILINGUAL CONSORTIUMS: EVALUATION OF THE COMMUNICATION TOOLS USED IN THE WANETAM NETWORK}

Marie Thorpe, ${ }^{1}$ Elizabeth Batchilly, ${ }^{1}$ Jean-Pierre N'Guessan, ${ }^{2}$

Dembo Kanteh'. 'MRC, The Gambia; ${ }^{1}$ University Hospital Centre Le Dantec, Senegal

\subsection{6/bmjgh-2016-000260.144}

Background Effective communication is a key challenge in managing multi-disciplinary teams (Bruce et al. 1995). This challenge is intensified when teams are dispersed in a multilingual consortium. Created in EDCTP's capacity building call, the "West African Network against TB, AIDS and Malaria" (WANETAM/WANETAM Plus) network partnered 19 research institutions from 10 West African countries. WANETAM's objectives are i) Capacity building and technology transfer to prepare West African sites for the successful conduct of clinical trials, ii) Creation of a network for subregional scientific collaborations. Whilst WANETAM successfully integrated capacity-building training, it is important to evaluate the effectiveness of the consortium's communication methods. This evaluation aims to i) Evaluate information sharing across a trilingual network; ii) Identify key successes, gaps in communications, iii) Identify tools that enable effective communication in multisite consortiums.

Methods Project documentation was reviewed to understand communication methods. An adapted Organizational Communication Audit questionnaire measured how communication systems aided desired project outputs/outcomes (Greenbaum et al 1988). Questions specifically evaluated the effectiveness of Basecamp, IP phones and email. This questionnaire was sent to WANETAM sites to gauge user perspectives.

Results Basecamp, the online collaboration project management software, enabled communications and document distribution in a single system. Despite efforts, usage was low. As predicted, technical difficulties with IP phones affected user acceptability. Email was the central communication method used to manage project milestones and deadlines. As the average person receives 121 emails daily over-reliance on email for primary communication poses challenges (Kane 2015).

Conclusions The role of e-collaboration is crucial in multi-site consortiums. Despite low usage, cloud computing networks remove the need for infrastructure (IP phones), lower costs and allow for accessibility regardless of location. When employed correctly, it achieves efficient, effective communication to achieve desired consortium objectives. 